\title{
Right atrial pressure alterations during echocardiography-guided-catheterization predict tricuspid valvular impairment: a novel method for the creation of a rabbit model of Staphylococcus aureus endocarditis
}

\author{
Mei-lian Wang ${ }^{1}$, Ying Zhang ${ }^{2}$, Miao Fan ${ }^{3}$ Ya-jun Guo², Wei-dong Ren² and En-jie Luo ${ }^{\text {1* }}$
}

\begin{abstract}
Background: We previously reported the use of a catheter system to damage the tricuspid valve and create infectious endocarditis (IE) in an animal model. The current study aims to create a faint IE model suitable for antibiotic prophylaxis using a low bacterial inoculum. We also aim to explore a way to quantitatively assess valvular impairment and to predict the success of the IE models during catheterization.

Methods: Ninety rabbits were assigned to two groups according to the density of bacteria inoculated $\left(1 \times 10^{5}\right.$ CFU for Group A and $1 \times 10^{4}$ CFU for Group B). A catheter system consisting of a polyethylene catheter and a guide wire were used to damage the valve. The catheter system was passed through the rabbits' tricuspid valves under echocardiographic guidance. A pressure transducer was used to assess right atrial pressure $\left(P_{R A}\right)$ before and just after valvular damage to calculate the pressure alterations $\left(\triangle \mathrm{P}_{\mathrm{RA}}\right)$. The animals in group $\mathrm{A}$ and $\mathrm{B}$ were divided into 3 subgroups according to the $\Delta P_{R A}\left(0-5 \mathrm{mmHg}\right.$ for Groups $A_{1}$ and $B_{1} ; 5-10 \mathrm{mmHg}$ for Groups $A_{2}$ and $B_{2}$; 10-15 mmHg for Groups $A_{3}$ and $B_{3}$ ). Staphylococcus aureus (ATCC 29213) inoculation was performed $24 \mathrm{hr}$ after cardiac catheterization.

Results: Faint IE was confirmed in $20 \%, 93.3 \%, 26.7 \%, 6.7 \%, 20 \%$, and $33.3 \%$ of the rabbits in Groups $A_{1}, A_{2}, A_{3}, B_{1}, B_{2}$, and $B_{3}$, respectively. There was no difference in the LV/RV ratio and $V_{T R}$ of the No-IE, faint-IE, and severe IE animals. Faint IE rabbits had a larger $\Delta P_{R A}$ than No-IE rabbits (7.81 \pm 1.21 vs. $2.48 \pm 1.0, P<0.01$, for Group $A ; 7.60 \pm 1.32$ vs. $2.98 \pm 1.08, P<0.01$, for Group B). The $\Delta P_{R A}$ of severe IE and faint IE rabbits was significantly different (13.11 \pm 1.31 vs. $7.81 \pm 1.21, P<0.01$, for Group A; $12.73 \pm 1.44$ vs.7.60 $\pm 1.32, P<0.01$, for Group B).

Conclusion: $\Delta P_{R A}$ could be used to assess valvular impairment. Controlling the value of $\Delta P_{R A}$ during catheterization and inoculating of an appropriate dose of bacteria was associated with a successful IE model.
\end{abstract}

Keywords: Infective endocarditis, Echocardiography, Catheterization, Right atrial pressure, Animal model

\footnotetext{
*Correspondence: enjieluo@outlook.com

'Department of Microbiology and Parasitology, College of Basic Medical Sciences, China Medical University, No. 92 Beier Road, Shenyang 110001, Heping District, China

Full list of author information is available at the end of the article
} 


\section{Background}

Infective endocarditis (IE) is a life-threatening disease associated with a high mortality rate [1-3]. It continues to be a challenge in clinical practice. Animal models of IE are widely used to provide a better understanding of the pathogenesis, pathophysiology [4-6], and treatment of intracardiac infections [7-9]. Normally, blood flows smoothly through cardiac valves. If these valves are damaged, the risk of bacterial attachment is increased. There are two main procedures used to create an IE model, damaging a valve and inoculating the host with bacteria. A large inoculum of bacteria is used for most IE models in order to guarantee the successful creation of IE [10-13]. Durack et al. injected $10^{8}$ colony-forming units (CFU) of viridans streptococci IV to produced IE in $100 \%$ of the experimental animals [14]. In fact, the dose of bacteria in humans may be 10 to 100 times higher than the lowest infecting dose necessary to produce IE in $90 \%$ of the animals. IE models created by injecting a large number of bacteria may be unsatisfactory as they may not be relevant to the human situation. For example, the magnitude of bacteremia observed in humans after certain procedures such as tooth extraction is generally $10^{1}$ to $10^{2} \mathrm{CFU} / \mathrm{ml}$ of blood [15]. A small inoculum similar to that found in humans with low-grade bacteremia is appropriate when the aim of the study is to evaluate the efficacy of a given antibiotic regimen given prior to bacterial challenge [16]. Using animal models with a large bacterial inoculum may require a prolonged administration of higher doses of antibiotics to achieve successful prophylaxis.

Small injected doses of bacteria may produce vegetations too small to be confirmed by echocardiography. This may be more relevant to the human situation [17-19]. We speculate that an appropriate aggravation for valvular damage might guarantee the successful creation of this IE model. We previously reported [20] a rabbit model of right-sided IE using a catheter to damage the tricuspid valve. This model is useful in evaluating the therapeutic effect of different medications on IE. We have improved this model to simulate the clinical setting of antibiotic prophylaxis. We reduced the size of the bacterial inoculation and evaluated valve impairment in this model of IE.

\section{Methods}

\section{Ethics statement}

All animal procedures were approved by the Animal Ethics Committee of China Medical University and were conducted in compliance with institutional regulations.

\section{Experimental design}

We speculated that the density of a bacterial inoculation can affect the development of IE. One hundred animals were used in the study. Ninety were randomly assigned to two groups according to the density of Staphylococcus aureus inoculated. $1 \times 10^{5} \mathrm{CFU}$ (Group A) or $1 \times 10^{4} \mathrm{CFU}$ (Group B) of S. aureus was inoculated $24 \mathrm{hr}$ after right heart catheterization. Five animals also had right heart catheterization performed without inoculation (Group C) and five animals were only inoculated with bacteria (Group D).

The extent of damage to the tricuspid valve affected the success rate of IE in the animal models. This might be related to the degree of right atrial pressure change related to instrumentation $\left(\Delta \mathrm{P}_{\mathrm{RA}}\right)$. We quantified valvular impairment by measuring the $\Delta \mathrm{P}_{\mathrm{RA}} . \Delta \mathrm{P}_{\mathrm{RA}}$ was controlled to a predicted range of values when damaging the tricuspid valves. For example, the catheter system was manipulated forward and backward across the tricuspid valve ten times to produce severe damage and once to produce mild damage. The number of times the guide wire was passed through the tricuspid valve led to different values of $\Delta \mathrm{P}_{\mathrm{RA}}$. Animals in Group $\mathrm{A}$ and $\mathrm{B}$ were divided into three sub-groups related to this effect of the wire on the pressure gradient across the tricuspid valve. The pressure gradients were $0-$ $5 \mathrm{mmHg}, 5-10 \mathrm{mmHg}$, and $10-15 \mathrm{mmHg}$, respectively for Groups $A_{1} / B_{1}, A_{2} / B_{2}$, and $A_{3} / B_{3}$. Each subgroup contained 15 animals. The ratio of left ventricle to right ventricle diameter (LV/RV), and peak velocity of tricuspid valve regurgitation $\left(\mathrm{V}_{\mathrm{TR}}\right)$ were evaluated during these procedures.

There were three outcomes in this animal model. Animals developed severe IE with vegetations visualized by echocardiography, faint IE with vegetations too small to be detected by echocardiography but confirmed by macroscopic and histologic examination of the cardiac valves, or did not develop IE by echocardiography or by histologic findings. The $\Delta \mathrm{P}_{\mathrm{RA}}$ and echocardiographic findings just after manipulating the tricuspid valves were used to assess model outcomes.

\section{Experimental animals}

One hundred New Zealand white rabbits (50 males and 50 females), weighing $2-2.5 \mathrm{~kg}$, were obtained from Beijing Animal Institute (Beijing, China). They were kept in the animal facility at China Medical University. The rabbits were maintained in individually ventilated cages and supplied heat-sterilized food and distilled water $a d$ libitum.

\section{Right heart catheterization under echocardiographic guidance}

Right heart catheterization was performed under echocardiographic guidance as previously described [20]. Briefly, an incision was made in the left inguinal region after induction of anesthesia with pentobarbital sodium 
(30 mg/Kg ip). The left femoral vein (LFV) was exposed and dissected to allow introduction of the catheter system. The catheter system consisted of a polyethylene catheter with a steel guide wire. The catheter system was prepared by flushing the external wall and the lumen of the catheter with heparinized sterile saline. The catheter system was introduced into the LFV to the entrance of the right atrium under echocardiographic guidance (Figure 1-A). When the catheter system touched the atrial septum, it was necessary to adjust the direction of the guide wire to advance the system pointing to the tricuspid valve (Figure 1-B) and then across it (Figure 1-C). The guide wire was advanced until about $1 \mathrm{~cm}$ of the tip was exposed. The guide wire was moved forward and backward repeatedly over the fragile tricuspid valve to induce damage.

\section{Evaluation of right atrial pressure $\left(\mathrm{P}_{\mathrm{RA}}\right)$}

The guide wire was slowly backed out after the catheter system was visualized entering the right atrium (Figure 1-A). A $20 \mathrm{ml}$ injection syringe (with $10 \mathrm{ml} \mathrm{sa-}$ line inside) was attached to the end of catheter using a three-way stopcock. Placement was checked using

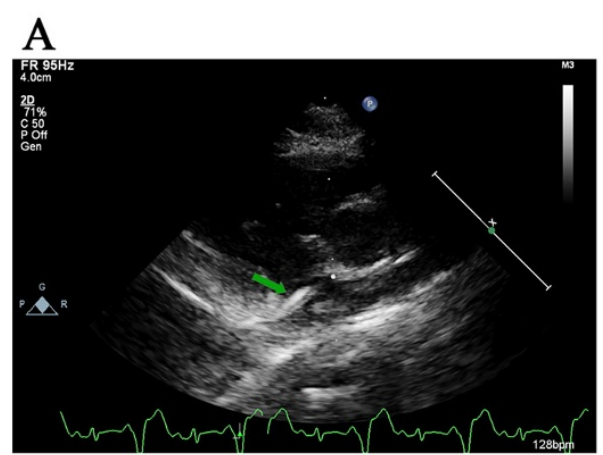

C
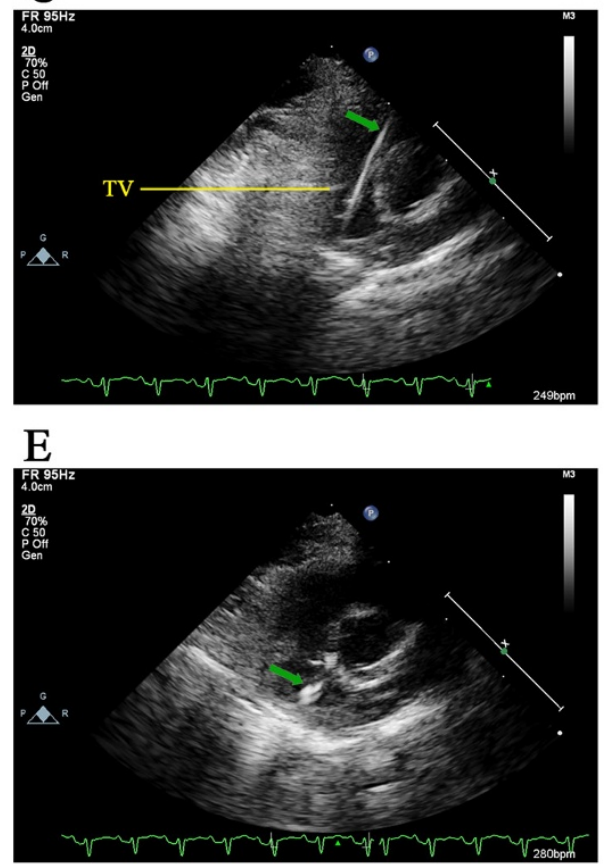

B

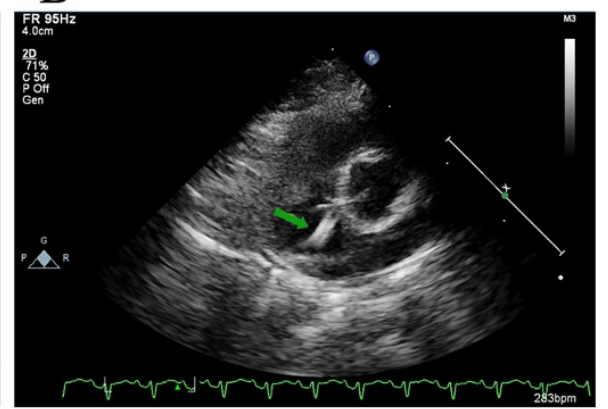

D

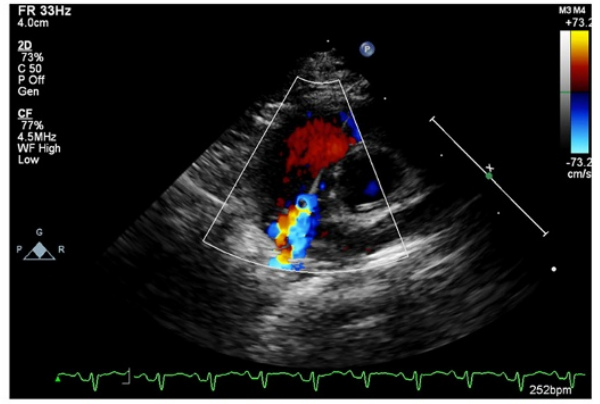

Figure 1 The process of right-heart catheterization under echocardiographic guidance. Echocardiography with an aortic short axis view was used to visualize the position of the catheter system. The catheter system was visualized entering the right atrium (A). The guide wire was slowly backed out to connect a pressure transducer to measure pressures in the right atrium. The pressure transducer was removed to allow introduction of the guide wire into the catheter system again. Adjust the direction of the guide wire to advance the catheter system pointing to the tricuspid valve (B) and then getting through it (C). The guide wire inside the catheter was then used to damage the tricuspid valve. Color Doppler was in action to visualize tricuspid valve regurgitation (D). The peak velocity was then evaluated by Pulsed Doppler. The catheter system was then repositioned at the entrance of the right atrium (E). The guide wire was removed and a pressure transducer connected to measure the pressure of the right atrium after valvular impairment. 
blood aspiration. A pressure transducer (YP-100, Yilian Medicine, Ltd., Shanghai, China) attached to the Biological and Functional Experimental System (BL-420, Taimeng Science technology, Ltd., Chengdu, China) was used to monitor pressure. Data was input into a personal computer using a USB 2.0 data cable. TM_WAVE Bio-signal acquisition and analysis software (version 1.0, Taimeng Science technology, Ltd., Chengdu, China) was used to display the $\mathrm{P}_{\mathrm{RA}}$ in real-time. Five randomly selected portions of the waveform were used to calculate the mean $\mathrm{P}_{\mathrm{RA}}$ (Figure 2-A). The pressure transducer was removed to allow introduction of the guide wire into the catheter system and to damage the tricuspid valve, which could be confirmed by the existence of tricuspid regurgitation by Color Doppler (Figure 1-D). The catheter system was then backed out into the right atrium (Figure 1-E). The guide wire was removed and the $\mathrm{P}_{\mathrm{RA}}$ monitored again (Figure 2-B).

\section{Echocardiographic measurement}

An ultrasound system (Philips iE 33) with a 4-12 MHz transducer was used in this study.
The heart was imaged using a four chamber view immediately after damaging the tricuspid valve. The diameters of the left and right ventricles were measured at the level of atrioventricular valve annulus and at the middle of the two ventricles. The average dimensions of the two ventricles were used to calculate the LV/RV ratio. A four chambers or aortic short axis view was used to confirm the existence of tricuspid valve regurgitation and to acquire the value of $\mathrm{V}_{\mathrm{TR}}$.

Echocardiography was performed to confirm the existence of cardiac vegetations on aortic short axis and four-chamber views when the rabbits were sacrificed or moribund.

\section{Production and confirmation of IE}

The rabbits were injected with a $S$. aureus (ATCC 29213) suspension $\left(1 \times 10^{5}\right.$ or $\left.1 \times 10^{4} \mathrm{CFU}\right)$ using a marginal ear vein. The body temperature was recorded during the challenge. Blood cultures were performed using intracardiac puncture at the end of the experiment. The presence of IE was confirmed by macroscopic and histologic examination of the cardiac valves. Tricuspid valves

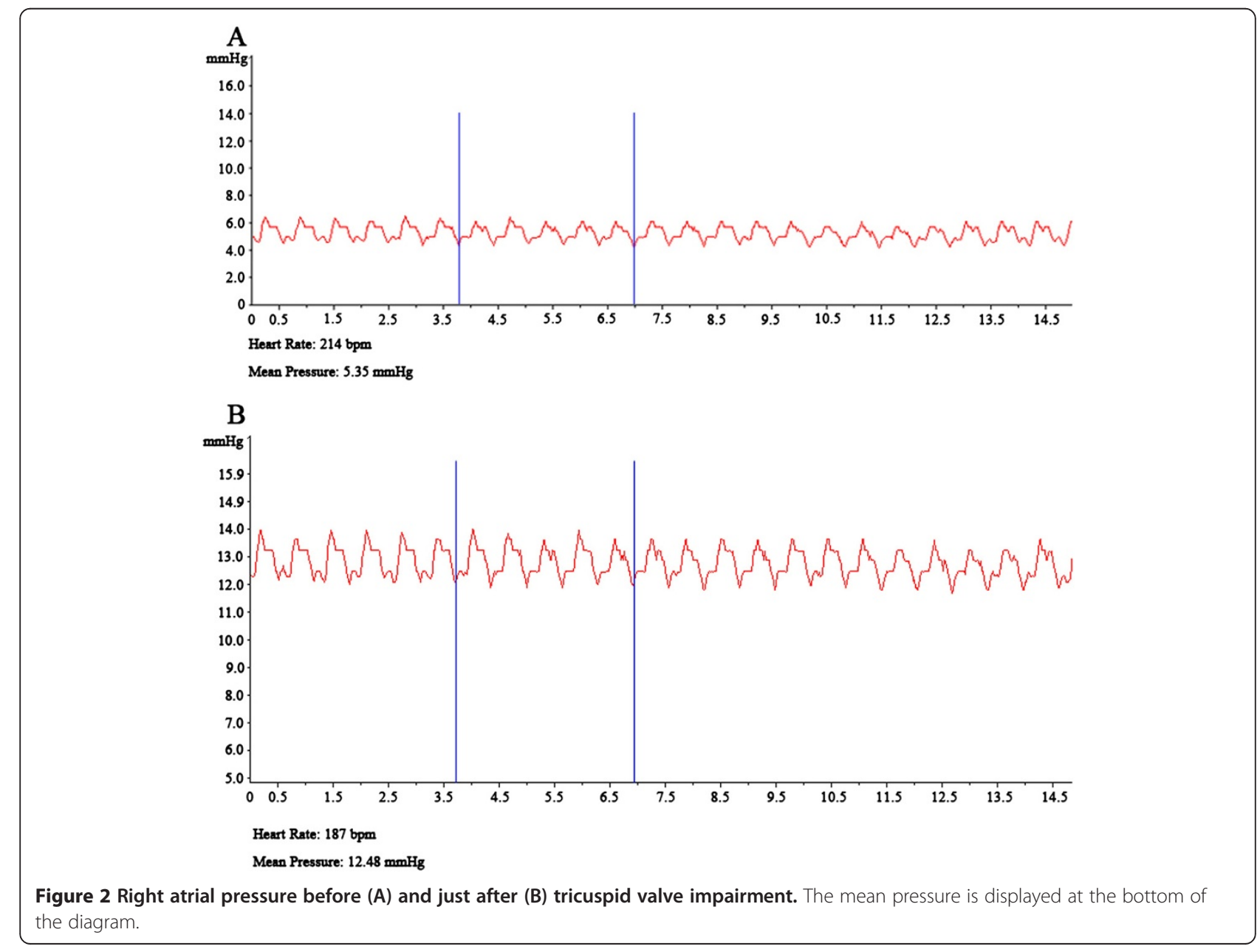


were excised and prepared for light microscopy. The specimens were fixed in 10\% formalin, embedded in paraffin and cut into $5 \mu \mathrm{m}$ sections. Sections were stained with hematoxylin \& eosin.

\section{Quantitative microbiologic analysis}

Bacterial titers per gram of tissue were determined for cardiac vegetations obtained from rabbits with IE. The tissue fragments were crushed in tryptic soy broth (Sigma, U.S.) and 10-fold serial dilutions were inoculated onto tryptic soy agar plates. Bacterial titers were reported in terms of CFU.

\section{Statistical analysis}

Data was expressed as means $\pm \mathrm{SD}$. Differences between multiple means were compared using one-way ANOVA, Tamhane's T2 test or Bonferroni test when the variance was heterogeneous or homogeneous, respectively. $P<0.05$ was considered statistically significant. All statistical analyses were performed using commercially available software (SPSS, release 17.0).

\section{Results}

Only one of the 90 rabbits in experimental groups A and B died prematurely. None of the control animals (Group C and D) died. The body temperature in all inoculated rabbits (Groups A, B, and D) increased to at least $40^{\circ} \mathrm{C}$ during the $72 \mathrm{hr}$ following inoculation. No discernible difference was observed between rabbits in Group A and B and no febrile response occurred in group C.

Macroscopic and histologic examination of the tricuspid valves demonstrated the presence of IE in 42 rabbits, 33 from Group A and 9 from Group B. Vegetations 1$10 \mathrm{~mm}$ in size were adherent to the tricuspid valve. The vegetations appeared yellow or gray-white. Histologic examination showed infectious vegetations with destruction of valvular tissue. Heavy inflammation composed mostly of neutrophils was present (Figure 3).

Blood cultures were positive in 29 rabbits from Group A and 3 rabbits from Group B. All except one were sacrificed on day 5 . The mean bacterial count of the cardiac vegetations was measured for each rabbit in Group A and B (Table 1).

Echocardiographic parameters and $\Delta \mathrm{P}_{\mathrm{RA}}$ were measured after damaging the tricuspid valve. The LV/RV ratio was about 2:1 in all the rabbits in Group A and B. There was no difference in the LV/RV ratio or $V_{T R}$ of the No-IE, Faint-IE, and Severe-IE subgroups. The $\Delta \mathrm{P}_{\mathrm{RA}}$ of the Faint IE rabbits was significantly higher than that of the No-IE rabbits $(P<0.01)$. The $\Delta \mathrm{P}_{\mathrm{RA}}$ of the Severe-IE rabbits was significantly higher than that of the Faint-IE rabbits $(P<0.01)$ (Table 2). The $\Delta \mathrm{P}_{\mathrm{RA}}$ during catheterization was able to predict the success of the IE models.

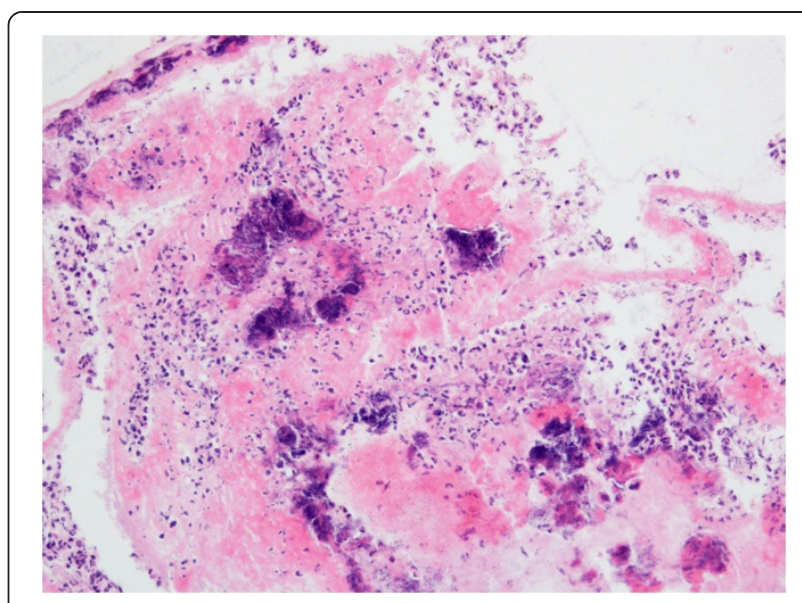

Figure 3 Histologic examination of the infected tricuspid valve. Histologic examination showed infectious vegetations with destruction of valvular tissue. Heavy inflammation consisting mostly of neutrophils was seen in the tissue (H\&E, 100X).

Animals in Groups A and B were divided into groups according to $\Delta \mathrm{P}_{\mathrm{RA}}$. Faint IE was confirmed in $20 \%$, $93.3 \%, 26.7 \%, 6.7 \%, 20 \%$, and $33.3 \%$ of the rabbits in Group $A_{1}, A_{2}, A_{3}, B_{1}, B_{2}$, and $B_{3}$, respectively (Table 3). The Faint-IE model best correlated with a $\Delta \mathrm{P}_{\mathrm{RA}}$ of $5-$ $10 \mathrm{mmHg}$ and an inoculation of $1 \times 10^{5} \mathrm{CFU}$ bacteria (Group $A_{2}$ ).

\section{Discussion}

IE animal models have been used to evaluate proposed changes in medical treatment. Cardiac valves are damaged and the animal inoculated with bacteria. The most primitive method was to damage cardiac valves by complex surgical procedures [21]. This modality was criticized because of the high mortality rate of the experimental animals. A catheter-related model for IE has been widely used since first introduced by Garrison and Freedoman [12] in 1970s. Usually, the catheter was used to destroy aortic valves when it was retrograded from carotid artery into ascending aorta [13]. However, it might cause

Table 1 Animal death, pathologic findings, blood culture results, and mean bacterial count in cardiac vegetations

\begin{tabular}{llllll}
\hline Experimental design & \multicolumn{2}{c}{ Experimental group } & & \multicolumn{2}{c}{ Control group } \\
\cline { 2 - 3 } & $\begin{array}{l}\text { Group A } \\
(\mathbf{n}=\mathbf{4 5})\end{array}$ & $\begin{array}{l}\text { Group B } \\
(\mathbf{n}=\mathbf{4 5})\end{array}$ & & $\begin{array}{l}\text { Group C } \\
(\mathbf{n}=\mathbf{5})\end{array}$ & $\begin{array}{l}\text { Group D } \\
(\mathbf{n}=\mathbf{5})\end{array}$ \\
\hline Premature death $(\mathrm{n})$ & 1 & 0 & 0 & 0 \\
Endocarditis $(\mathrm{n})$ & 33 & 9 & 0 & 0 \\
Positive blood culture $(\mathrm{n})$ & 29 & 3 & $\mathrm{~N} / \mathrm{A}$ & $\mathrm{N} / \mathrm{A}$ \\
MBC in vegetations $\left(\times 10^{4}\right)$ & $33.24 \pm 15.22$ & $9.78 \pm 5.66$ & N/A & N/A
\end{tabular}

Group A: rabbits underwent both catheterization and inoculation $\left(1 \times 10^{5} \mathrm{CFU}\right)$; Group B: rabbits underwent both catheterization and inoculation $\left(1 \times 10^{4} \mathrm{CFU}\right)$; Group C: rabbits underwent only catheterization; Group D: rabbits only inoculated. $M B C$ : Mean bacterial count; N/A: Not applicable. 
Table 2 Echocardiographic and physiological findings

\begin{tabular}{|c|c|c|c|c|c|c|}
\hline \multirow[t]{2}{*}{ Experimental design } & \multicolumn{3}{|c|}{ Group A $\left(1 \times 10^{5}\right.$ CFU) } & \multicolumn{3}{|c|}{ Group B $\left(1 \times 10^{4}\right.$ CFU $)$} \\
\hline & No-IE & Faint IE & Severe IE & No-IE & Faint IE & Severe IE \\
\hline Numbers & 12 & 21 & 12 & 36 & 9 & 0 \\
\hline LV/RV ratio & $2.05 \pm 0.23$ & $2.06 \pm 0.22$ & $1.94 \pm 0.22$ & $2.06 \pm 0.35$ & $2.01 \pm 0.18$ & $1.96 \pm 0.10$ \\
\hline$V_{T R}(m / s)$ & $0.53 \pm 0.26$ & $0.59 \pm 0.22$ & $0.66 \pm 0.24$ & $0.53 \pm 0.25$ & $0.58 \pm 0.21$ & $0.62 \pm 0.29$ \\
\hline$\Delta \mathrm{P}_{\mathrm{RA}}(\mathrm{mmHg})$ & $2.48 \pm 1.00$ & $7.81 \pm 1.21^{*}$ & $13.11 \pm 1.31^{\#}$ & $2.98 \pm 1.08$ & $7.6 \pm 1.32^{*}$ & $12.73 \pm 1.44^{\#}$ \\
\hline
\end{tabular}

No-IE: Did not develop IE by echocardiography or by histologic findings; Faint IE: Infectious endocarditis could confirmed by histologic findings but not visualized by echocardiography; Severe IE: Infectious endocarditis confirmed by both echocardiography and histologic findings. $L V$ : Left ventricle; $R V$ : Right ventricle; $V_{T R}$ : Peak velocity of tricuspid valve regurgitation; $\Delta P_{R A}$ : Right atrial pressure before and just after valvular impairment was used to calculate alterations of right atrial pressure, indicated as $\triangle \mathrm{P}_{\mathrm{RA}}$

${ }^{*} P<0.01$, Faint IE vs. None-IE; ${ }^{\#} P<0.01$, Severe IE vs. Faint IE.

relatively high mortality for the experimental animals. According to our experience, the heart rates of experimental animals such as rats or rabbits were very high. The retrograded catheter may severely damage aortic valves and then cause heart failure immediately. Many animals died within minutes after catheterization.

We developed a method [20] using a catheter system to damage the tricuspid valves under echocardiographic visualization. We reported the use of the catheter system with a guide wire inside to damage the tricuspid valves. We demonstrated a high survival rate and high infection rate with this reliable model. There were several limitations to this model. First, a high density of bacteria was used to ensure successful infection. This IE model was suited for therapeutic purposes, but not for prophylactic purposes. Second, the extent of tricuspid damage could not be quantitatively assessed when injuring the cardiac valves. Which animals were suitably infected could not be determined until several days after bacterial inoculation.

In the current study, we evaluated the use of different inoculum doses in the development of an IE model. Two doses of bacteria were inoculated. $1 \times 10^{5} \mathrm{CFU}$ was best suited for an IE prophylactic model. Previous studies have commonly used $1 \times 10^{6} \mathrm{CFU}$ [22] or even more [10-13]. The use of a relatively low density of inoculated bacteria is a highlight of this study.

Tricuspid valve regurgitation was confirmed in this new model just after damaging the tricuspid valves. There was not a difference in the $\mathrm{V}_{\mathrm{TR}}$ of No-IE, Faint-IE, and SevereIE animals. Inoculation density and valvular damage are the two main factors controlled in the development of an IE model. $V_{\text {TR }}$ was not a sensitive predictor of valvular impairment or the successful creation an IE model. Differences in $\Delta \mathrm{P}_{\mathrm{RA}}$ were related to valvular impairment and IE. The value of $\Delta \mathrm{P}_{\mathrm{RA}}$ was useful in guiding valvular damage to make the IE model. A faint-IE model was reliably created when $\Delta \mathrm{P}_{\mathrm{RA}}$ was kept between $5-10 \mathrm{mmHg}$. The faint-IE model is relevant in assessing the impact of medications on early stage IE. This right-sided IE model simulates human invasive diagnostic and therapeutic procedures, such as right heart catheter angiography, atrial septal defect occlusion, placement of intracardiac pacemakers [23].

In the current study, 90 animals were used to create IE models. $\Delta \mathrm{P}_{\mathrm{RA}}$ and inoculation dosage were used to evaluate the technique. Echocardiographic and physiologic parameters were also evaluated. The relatively large number of experimental animals and the detailed experimental design were strengths of this study. We found that control of $\Delta \mathrm{P}_{\mathrm{RA}}$ during catheterization and tricuspid valve damage was associated with the successful creation of IE, another highlight of our study. Our method is different from previous reports $[12,13]$ as the catheter system was removed immediately after catheterization. This modification is suitable for modeling intracardiac catheter procedures and the associated use of antibiotics $[11,24]$.

Table $3 \Delta \mathrm{P}_{\mathrm{RA}}$ and IE outcomes

\begin{tabular}{|c|c|c|c|c|c|c|}
\hline \multirow[t]{3}{*}{ Experimental design } & \multirow{2}{*}{\multicolumn{3}{|c|}{$\begin{array}{c}\text { Group A }(n=45) \\
1 \times 10^{5} \mathrm{CFU}\end{array}$}} & \multirow{2}{*}{\multicolumn{3}{|c|}{$\begin{array}{c}\text { Group B }(n=45) \\
1 \times 10^{4} \mathrm{CFU}\end{array}$}} \\
\hline & & & & & & \\
\hline & No-IE & Faint IE & Severe IE & No-IE & Faint IE & Severe IE \\
\hline$\Delta \mathrm{P}_{\mathrm{RA}}(0-5 \mathrm{mmHg})$ & $12 / 15(80 \%)$ & $3 / 15(20 \%)$ & $0 / 15(0 \%)$ & 14/15 (93.3\%) & $1 / 15(6.7 \%)$ & $0 / 15(0 \%)$ \\
\hline$\Delta \mathrm{P}_{\mathrm{RA}}(5-10 \mathrm{mmHg})$ & 0/15 (0\%) & 14/15 (93.3\%) & $1 / 15(6.7 \%)$ & $12 / 15(80 \%)$ & $3 / 15$ (20\%) & 0/15 (0\%) \\
\hline$\Delta \mathrm{P}_{\mathrm{RA}}(10-15 \mathrm{mmHg})$ & 0/15 (0\%) & $4 / 15(26.7 \%)$ & $11 / 15$ (73.3\%) & $10 / 15$ (66.7\%) & $5 / 15(33.3 \%)$ & 0/15 (0\%) \\
\hline
\end{tabular}

No-IE: Did not develop IE by echocardiography or by histologic findings; Faint IE: Infectious endocarditis could confirmed by histologic findings but not visualized by echocardiography; Severe IE: Infectious endocarditis confirmed by both echocardiography and histologic findings. $L V$ : Left ventricle; $R V$ : Right ventricle; $V_{T R}$ : Peak velocity of tricuspid valve regurgitation; $\Delta P_{R A}$ : Right atrial pressure before and just after valvular impairment was used to calculate alterations of right atrial pressure, indicated as $\Delta \mathrm{P}_{\mathrm{RA}}$. 
Compared with traditional techniques, we proposed a novel method to create an IE model in rabbits. A relatively low bacterial innoculum made this model more suitable for a prophylaxis purpose. In addition, echocardiographic guidance made it possible for the catheter systems precisely damage the valve. The most important point, we demonstrated that $\Delta \mathrm{P}_{\mathrm{RA}}$ could precisely assess valvular impairment and then predict the success of the IE models during catheterization. It could save lots of experimental animals using this novel method. Limitation of the study is the time of the procedures. Repeated measurements of $\Delta \mathrm{P}_{\mathrm{RA}}$ could prolong the procedures. For our study, the mean time for catheterization and measurement of $\Delta \mathrm{P}_{\mathrm{RA}}$ was $25 \pm 4 \mathrm{~min}$.

At last, we could read some additional message from the current study. $\mathrm{V}_{\mathrm{TR}}$ could not reflect the extent of valvular impairment just after injury, which indicates that $\mathrm{V}_{\mathrm{TR}}$ is not a sensitive index to reflect $\Delta \mathrm{P}_{\mathrm{RA}}$. Also, one may imagine that it is not always reliable to estimate pulmonary artery pressure using $\mathrm{V}_{\mathrm{TR}}$. For example, $\mathrm{V}_{\mathrm{TR}}$ is very low (tricuspid regurgitation could hardly be detected) while the pulmonary artery pressure is above $50 \mathrm{mmHg}$ measured by cardiac catheterization for a newborn.

\section{Conclusion}

We described a method to create a faint IE model using a relatively low bacterial inoculum. $\Delta \mathrm{P}_{\mathrm{RA}}$ was used to assess valvular impairment. Controlling the value of $\Delta \mathrm{P}_{\mathrm{RA}}$ during catheterization and inoculating of an appropriate dose of bacteria was associated with a successful IE model.

\section{Abbreviations}

CFU: Colony-forming units; IE: Infective endocarditis; LV: Left ventricle; $R_{P A}$ : Right atrial pressure; $\triangle R_{P A}$ : Alterations of right atrial pressure; RV: Right ventricle; $V_{T R}$ : Peak velocity of tricuspid valve regurgitation.

\section{Competing interests}

The authors declare that they have no competing interests.

\section{Authors' contributions}

M-IW and YZ designed the whole study. M-IW, YZ, MF, Y-jG, W-dR, and E-jL drafted the manuscript. YZ, Y-jG and M-IW performed right heart catheterization, echocardiographic examinations, and physiologic examinations, respectively. M-IW performed the microbiologic experiments. YZ and M-IW interpreted the results and analysed the data. All authors have read and approved the final manuscript.

\section{Acknowledgements}

We would like to convey our deepest thanks to Dr. Ying Zhang and Dr. Ya-jun Guo, for their great effort in performing all the echocardiographic examinations in the study. We also thank LetPub for its linguistic assistance during the preparation of this manuscript. Mei-lian Wang was supported by grant from a scientific research project (No. L2012287) sponsored by the Education Department of Liaoning Province, China, grant from Natural Science Foundation of Liaoning Province (No. 2013021010). En-jie Luo was supported by grant from the National Natural Science Foundation of China (No. 30972572)

\section{Author details}

'Department of Microbiology and Parasitology, College of Basic Medical Sciences, China Medical University, No. 92 Beier Road, Shenyang 110001, Heping District, China. ${ }^{2}$ Department of Sonography, Shengjing Hospital of China Medical University, No. 36 Sanhao Street, Shenyang 110004, Heping District, China. ${ }^{3}$ Department of Radiology, The First Affiliated Hospital of Sun Yat-sen University, Guangzhou 510080, China.

Received: 8 May 2014 Accepted: 18 June 2014

Published: 20 June 2014

\section{References}

1. Byramji A, Gilbert JD, Byard RW: Sudden death as a complication of bacterial endocarditis. Am J Forensic Med Pathol 2011, 32:140-2.

2. Thuny F, Hubert S, Tribouilloy C, Le Dolley Y, Casalta JP, Riberi A, Chevalier F, Rusinaru D, Malaquin D, Remadi JP, Ammar AB, Avierinos JF, Collart F, Raoult D, Habib G: Sudden death in patients with infective endocarditis: findings from a large cohort study. Int J Cardiol 2013, 162:129-32.

3. Manne MB, Shrestha NK, Lytle BW, Nowicki ER, Blackstone E, Gordon SM, Pettersson G, Fraser TG: Outcomes after surgical treatment of native and prosthetic valve infective endocarditis. Ann Thorac Surg 2012, 93:489-93.

4. Gutschik E: The Enterococcus endocarditis model in experimental animals and its relevance to human infection. $J$ Antimicrob Chemother 1993, 31(Suppl D):87-95.

5. Baddour LM, Christensen GD, Lowrance JH, Simpson WA: Pathogenesis of experimental endocarditis. Rev Infect Dis 1989, 11:452-63.

6. Baddour LM: Infectious endocarditis: new concepts in pathogenesis. Curr Opin Infect Dis 1999, 12:201-4.

7. Scheld WM: Therapy of streptococcal endocarditis: correlation of animal model and clinical studies. J Antimicrob Chemother 1987, 20(Suppl A):71-85.

8. Glauser MP, Francioli P: Relevance of animal models to the prophylaxis of infective endocarditis. J Antimicrob Chemother 1987, 20(Suppl A):87-93.

9. Blatter M, Francioli P: Endocarditis prophylaxis: from experimental models to human recommendation. Eur Heart J 1995, 16(Suppl B):107-9.

10. Maurin M, Lepidi H, La Scola B, Feuerstein M, Andre M, Pellissier JF, Raoult D: Guinea pig model for Staphylococcus aureus native valve endocarditis. Antimicrob Agents Chemother 1997, 41:1815-7.

11. Yersin B, Glauser MP, Guze PA, Guze LB, Freedman LR: Experimental Escherichia coli endocarditis in rats: roles of serum bactericidal activity and duration of catheter placement. Infect Immun 1988, 56:1273-80.

12. Garrison PK, Freedman LR: Experimental endocarditis I. Staphylococcal endocarditis in rabbits resulting from placement of a polyethylene catheter in the right side of the heart. Yale J Biol Med 1970, 42:394-410.

13. Perlman BB, Freedman LR: Experimental endocarditis. II. Staphylococcal infection of the aortic valve following placement of a polyethylene catheter in the left side of the heart. Yale J Biol Med 1971, 44:206-13.

14. Durack DT, Petersdorf RG: Chemotherapy of experimental streptococcal endocarditis. I. comparison of commonly recommended prophylactic regimens. J Clin Invest 1973, 52:592-8.

15. Everett ED, Hirschmann JV: Transient bacteremia and endocarditis prophylaxis. a review. Medicine (Baltimore) 1977, 56:61-77.

16. Carbon C: Animal models of endocarditis. Int J Biomed Comput 1994, 36:59-67.

17. San Román JA, Vilacosta I, López J, Revilla A, Arnold R, Sevilla T, Rollán MJ: Role of transthoracic and transesophageal echocardiography in right-sided endocarditis: one echocardiographic modality does not fit all. J Am Soc Echocardiogr 2012, 25:807-14.

18. San Román JA, Vilacosta I: Role of transesophageal echocardiography in right-sided endocarditis. Echocardiography 1995, 12:669-72.

19. Jiang SL, Li BJ, Zhang T, Ren CL, Wang Y, Chen TT, Gao CQ: Surgical treatment of isolated right-sided infective endocarditis. Tex Heart Inst J 2011, 38:639-42.

20. Wang ML, Zhang $Y$, Fan M, Guo YJ, Ren WD, Luo EJ: A rabbit model of right-sided Staphylococcus aureus endocarditis created with echocardiographic guidance. Cardiovasc Ultrasound 2013, 11:3. 
21. Highman B, Roshe J, Altland PD: Production of endocarditis with Staphylococcus aureus and Streptococcus mitis in dogs with aortic insufficiency. Circ Res 1956, 4:250-6.

22. La Scola B, Lepidi H, Maurin M, Raoult D: A guinea pig model for $Q$ fever endocarditis. J Infect Dis 1998, 178:278-81.

23. Thanavaro KL, Nixon JV: Endocarditis 2014: an update. Heart Lung. in press.

24. Héraïef $E$, Glauser MP, Freedman LR: Natural history of aortic valve endocarditis in rats. Infect Immun 1982, 37:127-31.

doi:10.1186/1476-7120-12-21

Cite this article as: Wang et al:: Right atrial pressure alterations during echocardiography-guided-catheterization predict tricuspid valvular impairment: a novel method for the creation of a rabbit model of Staphylococcus aureus endocarditis. Cardiovascular Ultrasound 2014 12:21.

\section{Submit your next manuscript to BioMed Central and take full advantage of:}

- Convenient online submission

- Thorough peer review

- No space constraints or color figure charges

- Immediate publication on acceptance

- Inclusion in PubMed, CAS, Scopus and Google Scholar

- Research which is freely available for redistribution 